Bull. Korean Math. Soc. 50 (2013), No. 1, pp. 217-231

http://dx.doi.org/10.4134/BKMS.2013.50.1.217

\title{
A FUBINI THEOREM FOR GENERALIZED ANALYTIC FEYNMAN INTEGRAL ON FUNCTION SPACE
}

\author{
Il Yong Lee, Jae Gil Choi, and Seung Jun Chang
}

\begin{abstract}
In this paper we establish a Fubini theorem for generalized analytic Feynman integral and $L_{1}$ generalized analytic Fourier-Feynman transform for the functional of the form

$$
F(x)=f\left(\left\langle\alpha_{1}, x\right\rangle, \ldots,\left\langle\alpha_{m}, x\right\rangle\right),
$$

where $\left\{\alpha_{1}, \ldots, \alpha_{m}\right\}$ is an orthonormal set of functions from $L_{a, b}^{2}[0, T]$. We then obtain several generalized analytic Feynman integration formulas involving generalized analytic Fourier-Feynman transforms.
\end{abstract}

\section{Introduction}

Let $C_{0}[0, T]$ denote one-parameter Wiener space; that is the space of realvalued continuous functions $x(t)$ on $[0, T]$ with $x(0)=0$. The concept of $L_{1}$ analytic Fourier-Feynman transform(FFT) was introduced by Brue in [1]. In [2], Cameron and Storvick introduced an $L_{2}$ analytic FFT on Wiener space. In [9], Johnson and Skoug developed an $L_{p}$ analytic FFT theory for $1 \leq p \leq 2$ which extended the results in $[1,2]$ and gave various relationships between the $L_{1}$ and the $L_{2}$ theories. In [7, 8], Huffman, Skoug and Storvick established Fubini theorems for various analytic Wiener and Feynman integrals. In [5], Chang and Lee extend the results of $[7,8]$ to a very general function space $C_{a, b}[0, T]$ and Banach algebra $\mathcal{S}\left(L_{a, b}^{2}[0, T]\right)$.

The function space $C_{a, b}[0, T]$ induced by a generalized Brownian motion was introduced by J. Yeh in [12] and was used extensively by Chang and Chung [3]. In this paper, we establish a Fubini theorem for generalized analytic Feynman integral and $L_{1}$ generalized analytic Fourier-Feynman transform. We then establish the generalized analytic Feynman integration formulas involving $L_{1}$ generalized analytic Fourier-Feynman transforms.

The stochastic process used in this paper as well as in $[3,4,5,12]$, is nonstationary in time, is subject to a drift $a(t)$, and can be used to explain the

Received July 21, 2011; Revised February 25, 2012.

2010 Mathematics Subject Classification. Primary 60J65, 28C20.

Key words and phrases. generalized Brownian motion process, generalized analytic Feynman integral, generalized analytic Fourier-Feynman transform, Fubini theorem. 
position of the Ornstein-Uhlenbeck process in an external force field [11]. However, when $a(t) \equiv 0$ and $b(t)=t$ on $[0, T]$, the general function space $C_{a, b}[0, T]$ reduces to the Wiener space $C_{0}[0, T]$.

\section{Definitions and preliminaries}

Let $D=[0, T]$ and let $(\Omega, \mathcal{B}, P)$ be a probability measure space. A realvalued stochastic process $Y$ on $(\Omega, \mathcal{B}, P)$ and $D$ is called a generalized Brownian motion process if $Y(0, \omega)=0$ almost everywhere and for $0=t_{0}<t_{1}<\cdots<$ $t_{n} \leq T$, the $n$-dimensional random vector $\left(Y\left(t_{1}, \omega\right), \ldots, Y\left(t_{n}, \omega\right)\right)$ is normally distributed with the density function

$$
\begin{aligned}
K(\vec{t}, \vec{\eta})= & \left((2 \pi)^{n} \prod_{j=1}^{n}\left(b\left(t_{j}\right)-b\left(t_{j-1}\right)\right)\right)^{-1 / 2} \\
& \cdot \exp \left\{-\frac{1}{2} \sum_{j=1}^{n} \frac{\left(\left(\eta_{j}-a\left(t_{j}\right)\right)-\left(\eta_{j-1}-a\left(t_{j-1}\right)\right)\right)^{2}}{b\left(t_{j}\right)-b\left(t_{j-1}\right)}\right\},
\end{aligned}
$$

where $\vec{\eta}=\left(\eta_{1}, \ldots, \eta_{n}\right), \eta_{0}=0, \vec{t}=\left(t_{1}, \ldots, t_{n}\right), a(t)$ is an absolutely continuous real-valued function on $[0, T]$ with $a(0)=0, a^{\prime}(t) \in L^{2}[0, T]$, and $b(t)$ is a strictly increasing, continuously differentiable real-valued function with $b(0)=$ 0 and $b^{\prime}(t)>0$ for each $t \in[0, T]$.

As explained in [13, pp. 18-20], $Y$ induces a probability measure $\mu$ on the measurable space $\left(\mathbb{R}^{D}, \mathcal{B}^{D}\right)$ where $\mathbb{R}^{D}$ is the space of all real-valued functions $x(t), \quad t \in D$, and $\mathcal{B}^{D}$ is the smallest $\sigma$-algebra of subsets of $\mathbb{R}^{D}$ with respect to which all the coordinate evaluation maps $e_{t}(x)=x(t)$ defined on $\mathbb{R}^{D}$ are measurable. The triple $\left(\mathbb{R}^{D}, \mathcal{B}^{D}, \mu\right)$ is a probability measure space. This measure space is called the function space induced by the generalized Brownian motion process $Y$ determined by $a(\cdot)$ and $b(\cdot)$.

We note that the generalized Brownian motion process $Y$ determined by $a(\cdot)$ and $b(\cdot)$ is a Gaussian process with mean function $a(t)$ and covariance function $r(s, t)=\min \{b(s), b(t)\}$. By Theorem 14.2 [13, p. 187], the probability measure $\mu$ induced by $Y$, taking a separable version, is supported by $C_{a, b}[0, T]$ (which is equivalent to the Banach space of continuous functions $x$ on $[0, T]$ with $x(0)=0$ under the sup norm). Hence $\left(C_{a, b}[0, T], \mathcal{B}\left(C_{a, b}[0, T]\right), \mu\right)$ is the function space induced by $Y$ where $\mathcal{B}\left(C_{a, b}[0, T]\right)$ is the Borel $\sigma$-algebra of $C_{a, b}[0, T]$.

Given two complex-valued measurable functions $F$ and $G$ on $C_{a, b}[0, T], F$ is said to be equal to $G$ for scale almost everywhere(s-a.e.) if for each $\rho>0$, $\mu\left(\left\{x \in C_{a, b}[0, T]: F(\rho x) \neq G(\rho x)\right\}\right)=0[6,10]$. We write that $F \approx G$ if $F=G$ for s-a.e..

Let $L_{a, b}^{2}[0, T]$ be the set of functions on $[0, T]$ which are Lebesgue measurable and square integrable with respect to the Lebesgue-Stieltjes measures on $[0, T]$ induced by $a(\cdot)$ and $b(\cdot)$; i.e.,

$$
L_{a, b}^{2}[0, T]=\left\{v: \int_{0}^{T} v^{2}(s) d b(s)<\infty \text { and } \int_{0}^{T} v^{2}(s) d|a|(s)<\infty\right\},
$$


where $|a|(t)$ denotes the total variation of the function $a(\cdot)$ on the interval $[0, t]$.

For $u, v \in L_{a, b}^{2}[0, T]$, let

$$
(u, v)_{a, b}=\int_{0}^{T} u(t) v(t) d[b(t)+|a|(t)] .
$$

Then $(\cdot, \cdot)_{a, b}$ is an inner product on $L_{a, b}^{2}[0, T]$ and $\|u\|_{a, b}=\sqrt{(u, u)_{a, b}}$ is a norm on $L_{a, b}^{2}[0, T]$. In particular, note that $\|u\|_{a, b}=0$ if and only if $u(t)=0$ a.e. on $[0, T]$. Furthermore, $\left(L_{a, b}^{2}[0, T],\|\cdot\|_{a, b}\right)$ is a separable Hilbert space.

Let $\left\{\phi_{j}\right\}_{j=1}^{\infty}$ be a complete orthogonal set of real-valued functions of bounded variation on $[0, T]$ such that

$$
\left(\phi_{j}, \phi_{k}\right)_{a, b}= \begin{cases}0, & j \neq k \\ 1, & j=k .\end{cases}
$$

Then for each $v \in L_{a, b}^{2}[0, T]$, the Paley-Wiener-Zygmund(PWZ) stochastic integral $\langle v, x\rangle$ is defined by the formula

$$
\langle v, x\rangle=\lim _{n \rightarrow \infty} \int_{0}^{T} \sum_{j=1}^{n}\left(v, \phi_{j}\right)_{a, b} \phi_{j}(t) d x(t)
$$

for all $x \in C_{a, b}[0, T]$ for which the limit exists.

Now, we state the definition of the generalized analytic Feynman integral.

Definition 2.1. Let $\mathbb{C}$ denote the complex numbers, let $\mathbb{C}_{+}=\{\lambda \in \mathbb{C}$ : $\operatorname{Re}(\lambda)>0\}$ and let $\tilde{\mathbb{C}}_{+}=\{\lambda \in \mathbb{C}: \lambda \neq 0$ and $\operatorname{Re}(\lambda) \geq 0\}$. Let $F: C_{a, b}[0, T] \rightarrow$ $\mathbb{C}$ be a measurable functional such that for each $\lambda>0$, the function space integral

$$
J(\lambda)=\int_{C_{a, b}[0, T]} F\left(\lambda^{-1 / 2} x\right) d \mu(x)
$$

exists. If there exists a function $J^{*}(\lambda)$ analytic in $\mathbb{C}_{+}$such that $J^{*}(\lambda)=J(\lambda)$ for all $\lambda>0$, then $J^{*}(\lambda)$ is defined to be the analytic function space integral of $F$ over $C_{a, b}[0, T]$ with parameter $\lambda$, and for $\lambda \in \mathbb{C}_{+}$we write

$$
J^{*}(\lambda)=\int_{C_{a, b}[0, T]}^{\mathrm{an}_{\lambda}} F(x) d \mu(x) .
$$

Let $q \neq 0$ be a real number and let $F$ be a functional such that

$$
\int_{C_{a, b}[0, T]}^{\mathrm{an}_{\lambda}} F(x) d \mu(x)
$$

exists for all $\lambda \in \mathbb{C}_{+}$. If the following limit exists, we call it the generalized analytic Feynman integral of $F$ with parameter $q$ and we write

$$
\int_{C_{a, b}[0, T]}^{\operatorname{anf}_{q}} F(x) d \mu(x)=\lim _{\lambda \rightarrow-i q} \int_{C_{a, b}[0, T]}^{\mathrm{an}_{\lambda}} F(x) d \mu(x),
$$

where $\lambda \rightarrow-i q$ through values in $\mathbb{C}_{+}$. 
The following notations are used throughout this paper:

$$
A_{\alpha_{j}} \equiv \int_{0}^{T} \alpha_{j}(t) d a(t) \quad \text { and } \quad B_{\alpha_{j}} \equiv \int_{0}^{T} \alpha_{j}^{2}(t) d b(t)
$$

for $\alpha_{j} \in L_{a, b}^{2}[0, T], j=1, \ldots, m$.

\section{A Fubini theorem for generalized analytic Feynman integral}

In this section we consider the functional of the form

$$
F(x)=f\left(\left\langle\alpha_{1}, x\right\rangle, \ldots,\left\langle\alpha_{m}, x\right\rangle\right),
$$

where $\left\{\alpha_{1}, \ldots, \alpha_{m}\right\}$ is an orthonormal set of functions from $L_{a, b}^{2}[0, T]$. We then establish a Fubini theorem for generalized analytic Feynman integral. Finally, we obtain several generalized analytic Feynman integration formulas.

In [5], the authors investigated several Fubini theorems involving the generalized Feynman integral and the generalized Fourier-Feynman transforms of functionals in the Banach algebra $\mathcal{S}\left(L_{a, b}^{2}[0, T]\right)$. The Banach algebra $\mathcal{S}\left(L_{a, b}^{2}[0, T]\right)$ consists of the functionals which are stochastic Fourier transforms of complexmeasures on $L_{a, b}^{2}[0, T]$. The results in [5] are characterized by the complexmeasures corresponding to the functionals in $\mathcal{S}\left(L_{a, b}^{2}[0, T]\right.$ ) (for instance, see $[5$, equation (3.12)]).

In this paper, we consider the cylinder functional $F$ given by equation (3.1). In fact, the class of all cylinder functionals forms a dense subset in $L^{2}\left(C_{a, b}[0, T]\right)$. In order to establish our Fubini theorem for the generalized analytic Feynman integral of the functional $F$ given by (3.1), we use a weight function (3.3) which is a cylinder functional.

Throughout the rest of this paper, we will use the following conventions: for given $q_{1}, \ldots, q_{n} \in \mathbb{R}-\{0\}$, let

$$
Q_{n}=\frac{q_{1} \cdots q_{n}}{\sum_{j=1}^{n} \frac{q_{1} \cdots q_{n}}{q_{j}}} \quad \text { where } \quad \sum_{j=1}^{k} \frac{q_{1} \cdots q_{k}}{q_{j}} \neq 0 \text { for all } k=2, \ldots, n
$$

and for simplicity, let

$$
\begin{gathered}
E_{q_{1}, \ldots, q_{n}}^{A_{\alpha_{j}}, B_{\alpha_{j}}}\left(\left\langle\alpha_{j}, \cdot\right\rangle\right)=\exp \left\{\left(-\frac{A_{\alpha_{j}}}{B_{\alpha_{j}}}+\frac{A_{\alpha_{j}} \sum_{j=1}^{n}\left(-i q_{j}\right)^{-1 / 2}}{B_{\alpha_{j}}\left(\sum_{j=1}^{n}\left(-i q_{j}\right)^{-1}\right)^{1 / 2}}\right)\left(-i Q_{n}\right)^{\frac{1}{2}}\left\langle\alpha_{j}, \cdot\right\rangle\right. \\
\left.-\frac{A_{\alpha_{j}}^{2} \sum_{1 \leq l<k \leq n}\left(-i q_{l}\right)^{-1 / 2}\left(-i q_{k}\right)^{-1 / 2}}{B_{\alpha_{j}} \sum_{j=1}^{n}\left(-i q_{j}\right)^{-1}}\right\}
\end{gathered}
$$

for $j=1, \ldots, m$.

We start this section by stating the following theorem which is a simple modification of [4, Theorem 4.1]. 
A FUBINI THEOREM FOR GENERALIZED ANALYTIC FEYNMAN INTEGRAL 221

Theorem 3.1. Let $q \in \mathbb{R}-\{0\}$ be given. Let $F$ be given by equation (3.1) with

$$
\int_{\mathbb{R}^{m}}|f(\vec{u})| \exp \left\{\left(\frac{1+|q|}{2}\right)^{\frac{1}{2}} \sum_{j=1}^{m} \frac{\left|A_{\alpha_{j}} u_{j}\right|}{B_{\alpha_{j}}}\right\} d \vec{u}<+\infty .
$$

Then the generalized analytic Feynman integral with parameter $q$ exists and is given by the formula

$$
\begin{aligned}
& \int_{C_{a, b}[0, T]}^{\operatorname{anf}_{q}} F(x) d \mu(x) \\
= & \left(\prod_{j=1}^{m} \frac{-i q}{2 \pi B_{\alpha_{j}}}\right)^{\frac{1}{2}} \int_{\mathbb{R}^{m}} f(\vec{u}) \exp \left\{-\sum_{j=1}^{m} \frac{\left[\sqrt{-i q} u_{j}-A_{\alpha_{j}}\right]^{2}}{2 B_{\alpha_{j}}}\right\} d \vec{u} .
\end{aligned}
$$

In our next theorem, we establish a Fubini theorem for the generalized analytic Feynman integrals.

Theorem 3.2. Let $q_{1}, q_{2} \in \mathbb{R}-\{0\}$. Let $F$ be given by equation (3.1) whose associated function $f$ satisfies the condition (3.4) with $|q|$ replaced with $\max \left\{\left|q_{1}\right|\right.$, $\left.\left|q_{2}\right|,\left|Q_{2}\right|\right\}$, where $Q_{2}$ is given by equation (3.2). Then

$$
\begin{aligned}
& \int_{C_{a, b}[0, T]}^{\operatorname{anf}_{q_{1}}} \int_{C_{a, b}[0, T]}^{\operatorname{anf}_{q_{2}}} F(x+y) d \mu(x) d \mu(y) \\
= & \int_{C_{a, b}[0, T]}^{\operatorname{anf}_{Q_{2}}} F(z) \prod_{j=1}^{m} E_{q_{1}, q_{2}}^{A_{\alpha_{j}}, B_{\alpha_{j}}}\left(\left\langle\alpha_{j}, z\right\rangle\right) d \mu(z) \\
= & \int_{C_{a, b}[0, T]}^{\operatorname{anf}_{q_{2}}} \int_{C_{a, b}[0, T]}^{\operatorname{anf}_{q_{1}}} F(x+y) d \mu(y) d \mu(x) .
\end{aligned}
$$

Also, all expressions in (3.6) are given by the expression

$$
\begin{aligned}
\left(\prod_{j=1}^{m} \frac{-i Q_{2}}{2 \pi B_{\alpha_{j}}}\right)^{\frac{1}{2}} \int_{\mathbb{R}^{m}} f(\vec{w}) \prod_{j=1}^{m} E_{q_{1}, q_{2}}^{A_{\alpha_{j}}, B_{\alpha_{j}}}\left(w_{j}\right) \\
\cdot \exp \left\{-\sum_{j=1}^{m} \frac{\left[\sqrt{-i Q_{2}} w_{j}-A_{\alpha_{j}}\right]^{2}}{2 B_{\alpha_{j}}}\right\} d \vec{w} .
\end{aligned}
$$

Proof. First, by using equations (3.1) and (3.5) with $q$ replaced with $q_{2}$, we obtain that for all $\lambda_{1}>0$

$$
\begin{aligned}
& \int_{C_{a, b}[0, T]} \int_{C_{a, b}[0, T]}^{\operatorname{anf}_{q_{2}}} F\left(x+\lambda_{1}^{-1 / 2} y\right) d \mu(x) d \mu(y) \\
= & \int_{C_{a, b}[0, T]}\left[\left(\prod_{j=1}^{m} \frac{-i q_{2}}{2 \pi B_{\alpha_{j}}}\right)^{\frac{1}{2}} \int_{\mathbb{R}^{m}} f\left(u_{1}+\lambda_{1}^{-1 / 2}\left\langle\alpha_{1}, y\right\rangle, \ldots, u_{m}+\lambda_{1}^{-1 / 2}\left\langle\alpha_{m}, y\right\rangle\right)\right.
\end{aligned}
$$




$$
\begin{aligned}
& \left.\cdot \exp \left\{-\sum_{j=1}^{m} \frac{\left[\sqrt{-i q_{2}} u_{j}-A_{\alpha_{j}}\right]^{2}}{2 B_{\alpha_{j}}}\right\} d \vec{u}\right] d \mu(y) \\
= & \left(\prod_{j=1}^{m} \frac{-i q_{2}}{2 \pi B_{\alpha_{j}}}\right)^{\frac{1}{2}}\left(\prod_{j=1}^{m} \frac{\lambda_{1}}{2 \pi B_{\alpha_{j}}}\right)^{\frac{1}{2}} \int_{\mathbb{R}^{m}} \int_{\mathbb{R}^{m}} f(\vec{u}+\vec{v}) \\
& \cdot \exp \left\{-\sum_{j=1}^{m} \frac{\left[\sqrt{-i q_{2}} u_{j}-A_{\alpha_{j}}\right]^{2}}{2 B_{\alpha_{j}}}-\sum_{j=1}^{m} \frac{\left[\sqrt{\lambda_{1}} v_{j}-A_{\alpha_{j}}\right]^{2}}{2 B_{\alpha_{j}}}\right\} d \vec{u} d \vec{v} .
\end{aligned}
$$

Let $u_{j}+v_{j}=w_{j}$ and $v_{j}=s_{j}$ for $j=1, \ldots, m$. Then we obtain that for all $\lambda_{1}>0$,

$$
\begin{aligned}
& \int_{C_{a, b}[0, T]} \int_{C_{a, b}[0, T]}^{\operatorname{anf}_{q_{2}}} F\left(x+\lambda_{1}^{-1 / 2} y\right) d \mu(x) d \mu(y) \\
= & \left(\prod_{j=1}^{m} \frac{-i q_{2}}{2 \pi B_{\alpha_{j}}}\right)^{\frac{1}{2}}\left(\prod_{j=1}^{m} \frac{\lambda_{1}}{2 \pi B_{\alpha_{j}}}\right)^{\frac{1}{2}} \int_{\mathbb{R}^{m}} \int_{\mathbb{R}^{m}} f(\vec{w}) \\
& \cdot \exp \left\{-\sum_{j=1}^{m} \frac{\left[\sqrt{-i q_{2}}\left(w_{j}-s_{j}\right)-A_{\alpha_{j}}\right]^{2}}{2 B_{\alpha_{j}}}-\sum_{j=1}^{m} \frac{\left[\sqrt{\lambda_{1}} s_{j}-A_{\alpha_{j}}\right]^{2}}{2 B_{\alpha_{j}}}\right\} d \vec{w} d \vec{s} .
\end{aligned}
$$

Carrying out the integration with respect to $s_{1}, \ldots, s_{m}$ in the above expression, we obtain

$$
\begin{aligned}
& \int_{C_{a, b}[0, T]} \int_{C_{a, b}[0, T]}^{\operatorname{anf}_{q_{2}}} F\left(x+\lambda_{1}^{-1 / 2} y\right) d \mu(x) d \mu(y) \\
= & \left(\prod_{j=1}^{m} \frac{-i q_{2}}{2 \pi B_{\alpha_{j}}}\right)^{\frac{1}{2}}\left(\prod_{j=1}^{m} \frac{\lambda_{1}}{2 \pi B_{\alpha_{j}}}\right)^{\frac{1}{2}} \int_{\mathbb{R}^{m}} f(\vec{w}) \exp \left\{-\sum_{j=1}^{m} \frac{\left[\sqrt{-i q_{2}} w_{j}-A_{\alpha_{j}}\right]^{2}}{2 B_{\alpha_{j}}}\right\} \\
& \cdot\left[\int _ { \mathbb { R } ^ { m } } \operatorname { e x p } \left\{-\sum_{j=1}^{m}\left(\frac{\left(-i q_{2}\right)+\lambda_{1}}{2 B_{\alpha_{j}}}\right) s_{j}^{2}\right.\right. \\
& \left.\left.+\sum_{j=1}^{m}\left(\frac{\sqrt{\lambda_{1}} A_{\alpha_{j}}+\sqrt{-i q_{2}}\left(\sqrt{-i q_{2}} w_{j}-A_{\alpha_{j}}\right)}{B_{\alpha_{j}}}\right) s_{j}+\sum_{j=1}^{m}\left(-\frac{A_{\alpha_{j}}^{2}}{2 B_{\alpha_{j}}}\right)\right\} d \vec{s}\right] d \vec{w} \\
= & \left(\prod_{j=1}^{m} \frac{1}{2 \pi B_{\alpha_{j}}}\right)^{\frac{1}{2}}\left(\frac{\left(-i q_{2}\right) \lambda_{1}}{\left(-i q_{2}\right)+\lambda_{1}}\right)^{\frac{m}{2}} \\
& \cdot \int_{\mathbb{R}^{m}} f(\vec{w}) \exp \left\{-\sum_{j=1}^{m} \frac{1}{2 B_{\alpha_{j}}}\left(\left(\frac{\left(-i q_{2}\right) \lambda_{1}}{\left(-i q_{2}\right)+\lambda_{1}}\right)^{\frac{1}{2}} w_{j}-A_{\alpha_{j}}\right)^{2}\right\} \\
& \cdot \exp \left\{\sum_{j=1}^{m}\left(-\frac{A_{\alpha_{j}}}{B_{\alpha_{j}}}+\frac{\left(\left(-i q_{2}\right)^{-1 / 2}+\lambda_{1}^{-1 / 2}\right) A_{\alpha_{j}}}{B_{\alpha_{j}} \sqrt{\left(-i q_{2}\right)^{-1}+\lambda_{1}^{-1}}}\right)\left(\frac{\left(-i q_{2}\right) \lambda_{1}}{\left(-i q_{2}\right)+\lambda_{1}}\right)^{\frac{1}{2}} w_{j}\right.
\end{aligned}
$$




$$
\left.-\sum_{j=1}^{m} \frac{\left(\left(-i q_{2}\right)^{-1 / 2} \lambda_{1}^{-1 / 2}\right) A_{\alpha_{j}}^{2}}{B_{\alpha_{j}}\left(\left(-i q_{2}\right)^{-1}+\lambda_{1}^{-1}\right)}\right\} d \vec{w} .
$$

Let

$$
\Gamma_{q_{1}}=\left\{\lambda_{1} \in \tilde{\mathbb{C}}_{+}: \operatorname{Re}\left(\lambda_{1}^{1 / 2}\right) \leq\left(\left(1+\left|q_{1}\right|\right) / 2\right)^{1 / 2}\right\}
$$

and let $J^{*}\left(\lambda_{1}\right)$ be a function given by the last expression of (3.9) for all $\lambda_{1} \in \Gamma_{q_{1}}$. Then $J^{*}$ is well-defined on the region $\Gamma_{q_{1}}$ and is an analytic function of $\lambda_{1}$ throughout the domain $\operatorname{Int}\left(\Gamma_{q_{1}}\right)$ so that $\int_{\Delta} J^{*}\left(\lambda_{1}\right) d \lambda_{1}=0$ for every rectifiable simple closed curve $\Delta$ lying in $\operatorname{Int}\left(\Gamma_{q_{1}}\right)$. Thus by using the Fubini theorem and Morera's theorem, the last expression of (3.9) above is an analytic function of $\lambda_{1}$ throughout the domain $\operatorname{Int}\left(\Gamma_{q_{1}}\right)$ and so letting $\lambda_{1} \rightarrow-i q_{1}$, we can see that the first expression of (3.6) is equal to the expression (3.7).

Next, using equation (3.5) with $q$ replaced with $Q_{2}$, we obtain that

$$
\begin{aligned}
& \int_{C_{a, b}[0, T]}^{\operatorname{anf}_{Q_{2}}} F(z) \prod_{j=1}^{m} E_{q_{1}, q_{2}}^{A_{\alpha_{j}}, B_{\alpha_{j}}}\left(\left\langle\alpha_{j}, z\right\rangle\right) d \mu(z) \\
= & \left(\prod_{j=1}^{m} \frac{-i Q_{2}}{2 \pi B_{\alpha_{j}}}\right)^{\frac{1}{2}} \int_{\mathbb{R}^{m}} f(\vec{w}) \prod_{j=1}^{m} E_{q_{1}, q_{2}}^{A_{\alpha_{j}}, B_{\alpha_{j}}}\left(w_{j}\right) \\
& \cdot \exp \left\{-\sum_{j=1}^{m} \frac{\left[\sqrt{-i Q_{2}} w_{j}-A_{\alpha_{j}}\right]^{2}}{2 B_{\alpha_{j}}}\right\} d \vec{w} .
\end{aligned}
$$

Finally, using (3.5) with $q$ replaced with $q_{1},(3.8)$ and (3.9) with $q_{2}$ replaced with $q_{1}$, respectively, and similar arguments as above, we can see that

$$
\begin{aligned}
& \int_{C_{a, b}[0, T]}\left[\int_{C_{a, b}[0, T]}^{\operatorname{anf}_{q_{1}}} F\left(\lambda_{2}^{-1 / 2} x+y\right) d \mu(y)\right] d \mu(x) \\
= & \left(\prod_{j=1}^{m} \frac{1}{2 \pi B_{\alpha_{j}}}\right)^{\frac{1}{2}}\left(\frac{\left(-i q_{1}\right) \lambda_{2}}{\left(-i q_{1}\right)+\lambda_{2}}\right)^{\frac{m}{2}} \\
& \cdot \int_{\mathbb{R}^{m}} f(\vec{w}) \exp \left\{-\sum_{j=1}^{m} \frac{1}{2 B_{\alpha_{j}}}\left(\left(\frac{\left(-i q_{1}\right) \lambda_{2}}{\left(-i q_{1}\right)+\lambda_{2}}\right)^{\frac{1}{2}} w_{j}-A_{\alpha_{j}}\right)^{2}\right\} \\
& \cdot \exp \left\{\sum _ { j = 1 } ^ { m } \left(-\frac{A_{\alpha_{j}}}{B_{\alpha_{j}}}+\frac{\left(\left(-i q_{1}\right)^{-1 / 2}+\lambda_{2}^{-1 / 2}\right) A_{\alpha_{j}}}{\left.B_{\alpha_{j}} \sqrt{\left(-i q_{1}\right)^{-1}+\lambda_{2}^{-1}}\right)\left(\frac{\left(-i q_{1}\right) \lambda_{2}}{\left(-i q_{1}\right)+\lambda_{2}}\right)^{\frac{1}{2}} w_{j}}\right.\right. \\
& \left.-\sum_{j=1}^{m} \frac{\left(\left(-i q_{1}\right)^{-1 / 2} \lambda_{2}^{-1 / 2}\right) A_{\alpha_{j}}^{2}}{B_{\alpha_{j}}\left(\left(-i q_{1}\right)^{-1}+\lambda_{2}^{-1}\right)}\right\} d \vec{w}
\end{aligned}
$$

is an analytic function of $\lambda_{2}$ through the domain $\operatorname{Int}\left(\Gamma_{q_{2}}\right)$ where $\Gamma_{q_{2}}=\left\{\lambda_{2} \in\right.$ $\left.\tilde{\mathbb{C}}_{+}: \operatorname{Re}\left(\lambda_{2}^{1 / 2}\right) \leq\left(\left(1+\left|q_{2}\right|\right) / 2\right)^{1 / 2}\right\}$, and so letting $\lambda_{2} \rightarrow-i q_{2}$, we can see that the third expression of (3.6) is equal to the expression (3.7). 
The condition (3.4) with $|q|$ replaced with $\max \left\{\left|q_{1}\right|,\left|q_{2}\right|,\left|Q_{2}\right|\right\}$ will ensure the existence of all expressions in (3.6) and (3.7).

Next corollary is easily obtained from Theorem 3.2.

Corollary 3.3. Let $F$ be as in Theorem 3.1. Then

$$
\begin{aligned}
& \int_{C_{a, b}[0, T]}^{\operatorname{anf}_{q}} \int_{C_{a, b}[0, T]}^{\operatorname{anf}_{q}} F(x+y) d \mu(x) d \mu(y) \\
= & \int_{C_{a, b}[0, T]}^{\operatorname{anf}_{q / 2}} F(z) \prod_{j=1}^{m} E_{q, q}^{A_{\alpha_{j}}, B_{\alpha_{j}}}\left(\left\langle\alpha_{j}, z\right\rangle\right) d \mu(z) .
\end{aligned}
$$

In our next theorem, we establish a Fubini theorem for the multiple generalized analytic Feynman integral.

Theorem 3.4. Let $q_{1}, \ldots, q_{n} \in \mathbb{R}-\{0\}$. Let $F$ be given by equation (3.1) whose associated function $f$ satisfies the condition (3.4) with $|q|$ replaced with $\max \left\{\left|q_{1}\right|, \ldots,\left|q_{n}\right|,\left|Q_{n}\right|\right\}$ where $Q_{n}$ is given by equation (3.2). Then

$$
\begin{aligned}
& \int_{C_{a, b}[0, T]}^{\operatorname{anf}_{q_{n}}} \cdots \int_{C_{a, b}[0, T]}^{\mathrm{anf}_{q_{1}}} F\left(x_{1}+\cdots+x_{n}\right) d \mu\left(x_{1}\right) \cdots d \mu\left(x_{n}\right) \\
= & \int_{C_{a, b}[0, T]}^{\operatorname{anf}_{Q_{n}}} F(z) \prod_{j=1}^{m} E_{q_{1}, \ldots, q_{n}}^{A_{\alpha_{j}}, B_{\alpha_{j}}}\left(\left\langle\alpha_{j}, z\right\rangle\right) d \mu(z) .
\end{aligned}
$$

Proof. By using equations (3.2), (3.3) and (3.6), we obtain inductively that

$$
\begin{aligned}
& \int_{C_{a, b}[0, T]}^{\operatorname{anf}_{q_{n}}} \cdots \int_{C_{a, b}[0, T]}^{\operatorname{anf}_{q_{1}}} F\left(x_{1}+\cdots+x_{n}\right) d \mu\left(x_{1}\right) \cdots d \mu\left(x_{n}\right) \\
= & \int_{C_{a, b}[0, T]}^{\operatorname{anf}_{q_{n}}} \cdots \int_{C_{a, b}[0, T]}^{\mathrm{anf}_{q_{3}}} \\
& \int_{C_{a, b}[0, T]}^{\operatorname{anf}_{Q_{2}}} F\left(y_{1}+x_{3}+\cdots+x_{n}\right) \prod_{j=1}^{m} E_{q_{1}, q_{2}}^{A_{\alpha_{j}, B_{\alpha_{j}}}}\left(\left\langle\alpha_{j}, y_{1}\right\rangle\right) d \mu\left(y_{1}\right) d \mu\left(x_{3}\right) \cdots d \mu\left(x_{n}\right) \\
= & \int_{C_{a, b}[0, T]}^{\operatorname{anf}_{q_{n}}} \cdots \int_{C_{a, b}[0, T]}^{\operatorname{anf}_{q_{4}}} \\
& \int_{C_{a, b}[0, T]}^{\operatorname{anf}_{Q_{3}}} F\left(y_{2}+x_{4}+\cdots+x_{n}\right) \prod_{j=1}^{m} E_{q_{1}, q_{2}, q_{3}}^{A_{\alpha_{j}}, B_{\alpha_{j}}}\left(\left\langle\alpha_{j}, y_{2}\right\rangle\right) d \mu\left(y_{2}\right) d \mu\left(x_{4}\right) \cdots d \mu\left(x_{n}\right) \\
= & \cdots \\
= & \int_{C_{a, b}[0, T]}^{\operatorname{anf}_{Q_{n}}} F(z) \prod_{j=1}^{m} E_{q_{1}, \ldots, q_{n}}^{A_{\alpha_{j}}, B_{\alpha_{j}}}\left(\left\langle\alpha_{j}, z\right\rangle\right) d \mu(z) .
\end{aligned}
$$

Thus we have the desired result. 
A FUBINI THEOREM FOR GENERALIZED ANALYTIC FEYNMAN INTEGRAL 225

Choosing $q_{j}=q$ for $j=1, \ldots, n$, we can easily obtain the following corollary of Theorem 3.4.

Corollary 3.5. Let $F$ be as in Theorem 3.1. Then

$$
\begin{aligned}
& \int_{C_{a, b}[0, T]}^{\operatorname{anf}_{q}} \cdots \int_{C_{a, b}[0, T]}^{\operatorname{anf}_{q}} F\left(x_{1}+\cdots+x_{n}\right) d \mu\left(x_{1}\right) \cdots d \mu\left(x_{n}\right) \\
= & \int_{C_{a, b}[0, T]}^{\operatorname{anf}_{q / n}} F(z) \prod_{j=1}^{m} E_{q, \ldots, q}^{A_{\alpha_{j}}, B_{\alpha_{j}}}\left(\left\langle\alpha_{j}, z\right\rangle\right) d \mu(z) .
\end{aligned}
$$

Lemma 3.6. Let $q_{0} \in \mathbb{R}-\{0\}$. Let $F$ be given by equation (3.1) with

$$
\int_{\mathbb{R}^{m}}|f(\vec{u})| \exp \left\{\left(\frac{1+\left|q_{0}\right|}{2}\right)^{\frac{1}{2}} \sum_{j=1}^{m} \frac{\left|A_{\alpha_{j}} u_{j}\right|}{B_{\alpha_{j}}}\right\} d \vec{u}<+\infty .
$$

Then for all real $q$ and $\gamma>0$ with $|\gamma q| \leq\left|q_{0}\right|$,

$$
\int_{C_{a, b}[0, T]}^{\operatorname{anf}_{\gamma q}} F(x) d \mu(x)=\int_{C_{a, b}[0, T]}^{\operatorname{anf}_{q}} F\left(\gamma^{-1 / 2} x\right) d \mu(x) .
$$

Proof. By using equation (3.5) with $q$ replaced with $\gamma q$, we have that

$$
\begin{aligned}
& \int_{C_{a, b}[0, T]}^{\operatorname{anf}_{\gamma q}} F(x) d \mu(x) \\
= & \left(\prod_{j=1}^{n} \frac{-i(\gamma q)}{2 \pi B_{\alpha_{j}}}\right)^{\frac{1}{2}} \int_{\mathbb{R}^{n}} f(\vec{u}) \exp \left\{-\sum_{j=1}^{n} \frac{\left[\sqrt{-i(\gamma q)} u_{j}-A_{\alpha_{j}}\right]^{2}}{2 B_{\alpha_{j}}}\right\} d \vec{u} \\
= & \left(\prod_{j=1}^{n} \frac{-i q}{2 \pi B_{\alpha_{j}}}\right)^{\frac{1}{2}} \int_{\mathbb{R}^{n}} f\left(\gamma^{-1 / 2} \vec{u}\right) \exp \left\{-\sum_{j=1}^{n} \frac{\left[\sqrt{-i q} u_{j}-A_{\alpha_{j}}\right]^{2}}{2 B_{\alpha_{j}}}\right\} d \vec{u} \\
= & \int_{C_{a, b}[0, T]}^{\operatorname{anf}_{q}} F\left(\gamma^{-1 / 2} x\right) d \mu(x) .
\end{aligned}
$$

Theorem 3.7. Let $F$ be as in Lemma 3.6. Then for all $q_{1}, q_{2} \in \mathbb{R}-\{0\}$ and $\gamma, \tau>0$ with $\left|q_{1} / \tau^{2}\right| \leq\left|q_{0}\right|,\left|q_{2} / \gamma^{2}\right| \leq\left|q_{0}\right|$ and $\left|q_{1} q_{2} /\left(\gamma^{2} q_{1}+\tau^{2} q_{2}\right)\right| \leq\left|q_{0}\right|$,

$$
\begin{aligned}
& \int_{C_{a, b}[0, T]}^{\operatorname{anf}_{q_{1}}} \int_{C_{a, b}[0, T]}^{\operatorname{anf}_{q_{2}}} F(\gamma x+\tau y) d \mu(x) d \mu(y) \\
= & \int_{C_{a, b}[0, T]}^{\operatorname{anf}_{q_{1} q_{2} /\left(\gamma^{2} q_{1}+\tau^{2} q_{2}\right)}} F(z) \prod_{j=1}^{m} E_{q_{2} / \gamma^{2}, q_{1} / \tau^{2}}^{A_{\alpha_{j}}, B_{\alpha_{j}}}\left(\left\langle\alpha_{j}, z\right\rangle\right) d \mu(z) .
\end{aligned}
$$


Proof. By using equations (3.6) and (3.11), we have that

$$
\begin{aligned}
& \int_{C_{a, b}[0, T]}^{\operatorname{anf}_{q_{1}}} \int_{C_{a, b}[0, T]}^{\operatorname{anf}_{q_{2}}} F(\gamma x+\tau y) d \mu(x) d \mu(y) \\
= & \int_{C_{a, b}[0, T]}^{\operatorname{anf}_{q_{1}}} \int_{C_{a, b}[0, T]}^{\operatorname{anf}_{q_{2} / \gamma^{2}}} F(x+\tau y) d \mu(x) d \mu(y) \\
= & \int_{C_{a, b}[0, T]}^{\operatorname{anf}_{q_{2} / \gamma^{2}}} \int_{C_{a, b}[0, T]}^{\operatorname{anf}_{q_{1}}} F(x+\tau y) d \mu(y) d \mu(x) \\
= & \int_{C_{a, b}[0, T]}^{\operatorname{anf}_{q_{2} / \gamma^{2}}} \int_{C_{a, b}[0, T]}^{\operatorname{anf}_{q_{1} / \tau^{2}}} F(x+y) d \mu(y) d \mu(x) \\
= & \int_{C_{a, b}[0, T]}^{\operatorname{anf}_{q_{1} q_{2} /\left(\gamma^{2} q_{1}+\tau^{2} q_{2}\right)}} F(z) \prod_{j=1}^{m} E_{q_{2} / \gamma^{2}, q_{1} / \tau^{2}}^{A_{\alpha_{j}}, B_{\alpha_{j}}}\left(\left\langle\alpha_{j}, z\right\rangle\right) d \mu(z) .
\end{aligned}
$$

Thus we have the desired result.

In the following examples, we exhibit functionals $F$ to apply our results above.

In Example 3.9 below, for simplicity, we will let $T=2$ and choose $a(t)=2 t$ and $b(t)=t^{2}+t$ on $[0,2]$. Next, let

$$
\alpha_{1}(t)= \begin{cases}\frac{1}{2}, & 0 \leq t \leq 1 \\ 0, & 1<t \leq 2 .\end{cases}
$$

Then, it follows easily that $\left\|\alpha_{1}\right\|_{a, b}=1, A_{\alpha_{1}}=1, B_{\alpha_{1}}=1 / 2$.

Example 3.8. Let $F: C_{a, b}[0,2] \rightarrow \mathbb{R}$ be defined by the formula

$$
F(x)=\exp \left\{-4\left\langle\alpha_{1}, x\right\rangle^{2}\right\} .
$$

By using equation (3.6) and a direct calculation, we obtain

$$
\begin{aligned}
& \int_{C_{a, b}[0, T]}^{\operatorname{anf}_{Q_{2}}} F(z) E_{q_{1}, q_{2}}^{1, \frac{1}{2}}\left(\left\langle\alpha_{1}, z\right\rangle\right) d \mu(z) \\
= & \left(\frac{-i Q_{2}}{\pi}\right)^{\frac{1}{2}} \int_{\mathbb{R}} E_{q_{1}, q_{2}}^{1, \frac{1}{2}}(w) \exp \left\{-4 w^{2}-\left[\sqrt{-i Q_{2}} w-1\right]^{2}\right\} d w
\end{aligned}
$$

and so

$$
\begin{aligned}
& \int_{C_{a, b}[0, T]}^{\operatorname{anf}_{q_{2}}} \int_{C_{a, b}[0, T]}^{\operatorname{anf}_{q_{1}}} F(x+y) d \mu(x) d \mu(y) \\
= & \left(\frac{-i Q_{2}}{\pi}\right)^{\frac{1}{2}} \int_{\mathbb{R}} E_{q_{1}, q_{2}}^{1, \frac{1}{2}}(w) \exp \left\{-4 w^{2}-\left[\sqrt{-i Q_{2}} w-1\right]^{2}\right\} d w .
\end{aligned}
$$

Let $\mathcal{M}\left(\mathbb{R}^{m}\right)$ denote the space of complex-valued Borel measures on $\mathcal{B}\left(\mathbb{R}^{m}\right)$. It is well known that a complex-valued Borel measure $\nu$ necessarily has a finite 
total variation $\|\nu\|$, and $\mathcal{M}\left(\mathbb{R}^{m}\right)$ is a Banach algebra under the norm $\|\cdot\|$ and with convolution as multiplication.

For $\nu \in \mathcal{M}\left(\mathbb{R}^{m}\right)$, the Fourier transform $\hat{\nu}$ of $\nu$ is a complex-valued function defined on $\mathbb{R}^{m}$ by the formula

$$
\hat{\nu}(\vec{u})=\int_{\mathbb{R}^{m}} \exp \left\{i \sum_{j=1}^{m} u_{j} v_{j}\right\} d \nu(\vec{v}),
$$

where $\vec{u}=\left(u_{1}, \ldots, u_{m}\right)$ and $\vec{v}=\left(v_{1}, \ldots, v_{m}\right)$ are in $\mathbb{R}^{m}$.

Example 3.9. Let $\left\{\alpha_{1}, \ldots, \alpha_{m}\right\}$ be an orthonormal set of functions from $L_{a, b}^{2}[0, T]$. Define the functional $F: C_{a, b}[0, T] \rightarrow \mathbb{C}$ by

$$
F(x)=\hat{\nu}\left(\left\langle\alpha_{1}, x\right\rangle, \ldots,\left\langle\alpha_{m}, x\right\rangle\right),
$$

where $\hat{\nu}$ is the Fourier transform of $\nu$ in $\mathcal{M}\left(\mathbb{R}^{m}\right)$.

Now by using equation (3.6) and a direct calculation, we obtain

$$
\begin{aligned}
& \int_{C_{a, b}[0, T]}^{\operatorname{anf}_{Q_{2}}} F(z) \prod_{j=1}^{m} E_{q_{1}, q_{2}}^{A_{\alpha_{j}}, B_{\alpha_{j}}}\left(\left\langle\alpha_{j}, z\right\rangle\right) d \mu(z) \\
= & \int_{\mathbb{R}^{m}} \exp \left\{-\frac{i}{2 Q_{2}} \sum_{j=1}^{m} B_{\alpha_{j}} v_{j}^{2}+i\left(\frac{i}{Q_{2}}\right)^{\frac{1}{2}} \frac{\sqrt{-i q_{1}}+\sqrt{-i q_{2}}}{\sqrt{\left(-i q_{1}\right)+\left(-i q_{2}\right)}} \sum_{j=1}^{m} A_{\alpha_{j}} v_{j}\right\} d \nu(\vec{v})
\end{aligned}
$$

and hence

$$
\begin{aligned}
& \int_{C_{a, b}[0, T]}^{\operatorname{anf}_{q_{2}}} \int_{C_{a, b}[0, T]}^{\operatorname{anf}_{q_{1}}} F(x+y) d \mu(x) d \mu(y) \\
= & \int_{\mathbb{R}^{m}} \exp \left\{-\frac{i}{2 Q_{2}} \sum_{j=1}^{m} B_{\alpha_{j}} v_{j}^{2}+i\left(\frac{i}{Q_{2}}\right)^{\frac{1}{2}} \frac{\sqrt{-i q_{1}}+\sqrt{-i q_{2}}}{\sqrt{\left(-i q_{1}\right)+\left(-i q_{2}\right)}} \sum_{j=1}^{m} A_{\alpha_{j}} v_{j}\right\} d \nu(\vec{v}) .
\end{aligned}
$$

\section{An $L_{1}$ generalized analytic Fourier-Feynman transforms}

In Section 3 above, we studied a Fubini theorem for generalized analytic Feynman integral. In this section, we establish a Fubini theorem for $L_{1}$ generalized analytic Fourier-Feynman transform(GFFT). We then establish the generalized analytic Feynman integration formulas involving $L_{1}$ analytic GFFT.

We state the definition of the $L_{1}$ analytic GFFT.

Definition 4.1. For $\lambda \in \mathbb{C}_{+}$and $y \in C_{a, b}[0, T]$, let

$$
T_{\lambda}(F)(y)=\int_{C_{a, b}[0, T]}^{\mathrm{an}_{\lambda}} F(y+x) d \mu(x) .
$$

Then for $q \in \mathbb{R}-\{0\}$, the $L_{1}$ analytic GFFT, $T_{q}^{(1)}(F)$ of $F$ is defined by the formula $\left(\lambda \in \mathbb{C}_{+}\right)$

$$
T_{q}^{(1)}(F)(y)=\lim _{\lambda \rightarrow-i q} T_{\lambda}(F)(y)
$$


for s-a.e. $y \in C_{a, b}[0, T]$ whenever the limit exists. That is to say,

$$
T_{q}^{(1)}(F)(y)=\int_{C_{a, b}[0, T]}^{\operatorname{anf}_{q}} F(y+x) d \mu(x)
$$

for s-a.e. $y \in C_{a, b}[0, T]$.

We note that if $T_{q}^{(1)}(F)$ exists and if $F \approx G$, then $T_{q}^{(1)}(G)$ exists and $T_{q}^{(1)}(G) \approx T_{q}^{(1)}(F)$.

Now we present our results for the $L_{1}$ analytic GFFT and multiple $L_{1}$ analytic GFFT for the functional $F$ without proof.

Theorem 4.2. Let $q$ and $F$ be as in Theorem 3.1. Then the $L_{1}$ analytic GFFT of $F, T_{q}^{(1)}(F)$ exists and is given by the formula

$$
\begin{aligned}
T_{q}^{(1)}(F)(y)= & \left(\prod_{j=1}^{m} \frac{-i q}{2 \pi B_{\alpha_{j}}}\right)^{\frac{1}{2}} \int_{\mathbb{R}^{m}} f(\vec{u}+\langle\vec{\alpha}, y\rangle) \\
& \cdot \exp \left\{-\sum_{j=1}^{m} \frac{\left[\sqrt{-i q} u_{j}-A_{\alpha_{j}}\right]^{2}}{2 B_{\alpha_{j}}}\right\} d \vec{u}
\end{aligned}
$$

for s-a.e. $y \in C_{a, b}[0, T]$.

Theorem 4.3. Let $q_{1}, \ldots, q_{n}$ and $F$ be as in Theorem 3.4. Then the multiple $L_{1}$ analytic GFFT of $F, T_{q_{n}}^{(1)}\left(T_{q_{n-1}}^{(1)}\left(\cdots\left(T_{q_{2}}^{(1)}\left(T_{q_{1}}^{(1)}(F)\right)\right) \cdots\right)\right)$ exists and is given by the formula

$$
\begin{aligned}
& T_{q_{n}}^{(1)}\left(T_{q_{n-1}}^{(1)}\left(\cdots\left(T_{q_{2}}^{(1)}\left(T_{q_{1}}^{(1)}(F)\right)\right) \cdots\right)\right)(y) \\
= & \prod_{l=1}^{n}\left(\prod_{j=1}^{m} \frac{-i q_{l}}{2 \pi B_{\alpha_{j}}}\right)^{\frac{1}{2}} \int_{\mathbb{R}^{m}} \cdots \int_{\mathbb{R}^{m}} f\left(\vec{u}_{1}+\cdots+\vec{u}_{n}+\langle\vec{\alpha}, y\rangle\right) \\
& \cdot \exp \left\{-\sum_{j=1}^{m} \frac{\left[\sqrt{-i q_{1}} u_{1 j}-A_{\alpha_{j}}\right]^{2}}{2 B_{\alpha_{j}}}-\cdots-\sum_{j=1}^{m} \frac{\left[\sqrt{-i q_{n}} u_{n j}-A_{\alpha_{j}}\right]^{2}}{2 B_{\alpha_{j}}}\right\} d \vec{u}_{1} \cdots d \vec{u}_{n}
\end{aligned}
$$

for $s$-a.e. $y \in C_{a, b}[0, T]$, where $\vec{u}_{1}=\left(u_{11}, \ldots, u_{1 m}\right), \ldots, \vec{u}_{n}=\left(u_{n 1}, \ldots, u_{n m}\right) \in$ $\mathbb{R}^{m}$.

In our next theorem, we establish a formula for the generalized analytic Feynman integral involving the $L_{1}$ analytic GFFT.

Theorem 4.4. Let $q_{0}$ and $F$ be as in Lemma 3.6. Then for all $q_{1}, q_{2} \in \mathbb{R}-\{0\}$ and all $\gamma>0$ with $\left|\gamma q_{j}\right| \leq\left|q_{0}\right|$ for $j=1,2$,

$$
\begin{aligned}
\int_{C_{a, b}[0, T]}^{\operatorname{anf}_{\gamma q_{2}}}\left(T_{q_{1}}^{(1)}(F)\right)(\sqrt{\gamma} y) d \mu(y) & =\int_{C_{a, b}[0, T]}^{\operatorname{anf}_{Q_{2}}} F(z) \prod_{j=1}^{m} E_{q_{1}, q_{2}}^{A_{\alpha_{j}}, B_{\alpha_{j}}}\left(\left\langle\alpha_{j}, z\right\rangle\right) d \mu(z) \\
& =\int_{C_{a, b}[0, T]}^{\operatorname{anf}_{\gamma q_{1}}}\left(T_{q_{2}}^{(1)}(F)\right)(\sqrt{\gamma} x) d \mu(x) .
\end{aligned}
$$


Proof. By using equations (3.6), (3.11) and (4.1), we have that

$$
\begin{aligned}
& \int_{C_{a, b}[0, T]}^{\operatorname{anf}_{\gamma q_{2}}}\left(T_{q_{1}}^{(1)}(F)\right)(\sqrt{\gamma} y) d \mu(y) \\
= & \int_{C_{a, b}[0, T]}^{\operatorname{anf}_{\gamma q_{2}}} \int_{C_{a, b}[0, T]}^{\operatorname{anf}_{q_{1}}} F(\sqrt{\gamma} y+x) d \mu(x) d \mu(y) \\
= & \int_{C_{a, b}[0, T]}^{\operatorname{anf}_{q_{1}}} \int_{C_{a, b}[0, T]}^{\operatorname{anf}_{\gamma q_{2}}} F(\sqrt{\gamma} y+x) d \mu(y) d \mu(x) \\
= & \int_{C_{a, b}[0, T]}^{\operatorname{anf}_{q_{1}}} \int_{C_{a, b}[0, T]}^{\operatorname{anf}_{q_{2}}} F(y+x) d \mu(y) d \mu(x) \\
= & \int_{C_{a, b}[0, T]}^{\operatorname{anf}_{Q_{2}}} F(z) \prod_{j=1}^{m} E_{q_{1}, q_{2}}^{A_{\alpha_{j}}, B_{\alpha_{j}}}\left(\left\langle\alpha_{j}, z\right\rangle\right) d \mu(z) .
\end{aligned}
$$

Also, by using the similar method used in (4.3), we obtain that

$$
\int_{C_{a, b}[0, T]}^{\operatorname{anf}_{\gamma q_{1}}}\left(T_{q_{2}}^{(1)}(F)\right)(\sqrt{\gamma} x) d \mu(x)=\int_{C_{a, b}[0, T]}^{\operatorname{anf}_{Q_{2}}} F(z) \prod_{j=1}^{m} E_{q_{1}, q_{2}}^{A_{\alpha_{j}}, B_{\alpha_{j}}}\left(\left\langle\alpha_{j}, z\right\rangle\right) d \mu(z) .
$$

Thus we have the desired result.

Our next corollary below says that the generalized analytic Feynman integral with parameter $q_{2}$ of the $L_{1}$ analytic GFFT with parameter $q_{1}$ equals the generalized analytic Feynman integral with parameter $q_{1}$ of the $L_{1}$ analytic GFFT with parameter $q_{2}$.

Corollary 4.5. Let $q_{0}$ and $F$ be as in Lemma 3.6. Then for all $q_{1}, q_{2} \in \mathbb{R}-\{0\}$ with $\left|q_{j}\right| \leq\left|q_{0}\right|$ for $j=1,2$,

$$
\begin{aligned}
\int_{C_{a, b}[0, T]}^{\operatorname{anf}_{q_{2}}}\left(T_{q_{1}}^{(1)}(F)\right)(y) d \mu(y) & =\int_{C_{a, b}[0, T]}^{\operatorname{anf}_{Q_{2}}} F(z) \prod_{j=1}^{m} E_{q_{1}, q_{2}}^{A_{\alpha_{j}}, B_{\alpha_{j}}}\left(\left\langle\alpha_{j}, z\right\rangle\right) d \mu(z) \\
& =\int_{C_{a, b}[0, T]}^{\operatorname{anf}_{q_{1}}}\left(T_{q_{2}}^{(1)}(F)\right)(x) d \mu(x) .
\end{aligned}
$$

Also, choosing $q_{j}=q$ for $j=1,2$,

$$
\int_{C_{a, b}[0, T]}^{\operatorname{anf}_{q}}\left(T_{q}^{(1)}(F)\right)(y) d \mu(y)=\int_{C_{a, b}[0, T]}^{\operatorname{anf}_{q / 2}} F(z) \prod_{j=1}^{m} E_{q, q}^{A_{\alpha_{j}}, B_{\alpha_{j}}}\left(\left\langle\alpha_{j}, z\right\rangle\right) d \mu(z) .
$$

In our next theorem, we establish a formula for the multiple $L_{1}$ analytic GFFT. 
Theorem 4.6. Let $q_{1}, \ldots, q_{n}$ and $F$ be as in Theorem 3.4. Then

$$
\begin{aligned}
& T_{q_{n}}^{(1)}\left(T_{q_{n-1}}^{(1)}\left(\cdots\left(T_{q_{2}}^{(1)}\left(T_{q_{1}}^{(1)}(F)\right)\right) \cdots\right)\right)(y) \\
= & \int_{C_{a, b}[0, T]}^{\operatorname{anf} Q_{Q_{n}}} F(y+x) \prod_{j=1}^{m} E_{q_{1}, \ldots, q_{n}}^{A_{\alpha_{j}}, B_{\alpha_{j}}}\left(\left\langle\alpha_{j}, x\right\rangle\right) d \mu(x)
\end{aligned}
$$

for s-a.e. $y \in C_{a, b}[0, T]$. In addition, both expressions in (4.4) are given by the right-hand side of equation (4.2) above.

Proof. By using equations (3.10) and (4.1), we have

$$
\begin{aligned}
& T_{q_{n}}^{(1)}\left(T_{q_{n-1}}^{(1)}\left(\cdots\left(T_{q_{2}}^{(1)}\left(T_{q_{1}}^{(1)}(F)\right)\right) \cdots\right)\right)(y) \\
= & \int_{C_{a, b}[0, T]}^{\operatorname{anf}_{q_{n}}} \cdots \int_{C_{a, b}[0, T]}^{\operatorname{anf}_{q_{1}}} F\left(y+z_{1}+\cdots+z_{n}\right) d \mu\left(z_{1}\right) \cdots d \mu\left(z_{n}\right) \\
= & \int_{C_{a, b}[0, T]}^{\operatorname{anf}_{Q_{n}}} F(y+x) \prod_{j=1}^{m} E_{q_{1}, \ldots, q_{n}}^{A_{\alpha_{j}}, B_{\alpha_{j}}}\left(\left\langle\alpha_{j}, x\right\rangle\right) d \mu(x)
\end{aligned}
$$

for s-a.e. $y \in C_{a, b}[0, T]$.

Choosing $q_{j}=q$ for $j=1, \ldots, n$, we can easily obtain the following corollary.

Corollary 4.7. Let $q$ and $F$ be as in Theorem 4.2. Then

(i) for s-a.e. $y \in C_{a, b}[0, T]$,

$$
\left(T_{q}^{(1)}\left(T_{q}^{(1)}(F)\right)\right)(y)=\int_{C_{a, b}[0, T]}^{\mathrm{anf}_{q / 2}} F(x+y) \prod_{j=1}^{m} E_{q, q}^{A_{\alpha_{j}}, B_{\alpha_{j}}}\left(\left\langle\alpha_{j}, x\right\rangle\right) d \mu(x),
$$

and

(ii) for s-a.e. $y \in C_{a, b}[0, T]$,

$$
\begin{aligned}
& T_{q}^{(1)}\left(T_{q}^{(1)}\left(\cdots\left(T_{q}^{(1)}\left(T_{q}^{(1)}(F)\right)\right) \cdots\right)\right)(y) \\
= & \int_{C_{a, b}[0, T]}^{\mathrm{anf}_{q / n}} F(y+x) \prod_{j=1}^{m} E_{q, \ldots, q}^{A_{\alpha_{j}}, B_{\alpha_{j}}}\left(\left\langle\alpha_{j}, x\right\rangle\right) d \mu(x) .
\end{aligned}
$$

Corollary 4.8. Let $q_{1}, q_{2}$ and $F$ be as in Theorem 3.2. Then

$$
\begin{aligned}
T_{q_{2}}^{(1)}\left(T_{q_{1}}^{(1)}(F)\right)(y) & =\int_{C_{a, b}[0, T]}^{\operatorname{anf}_{Q_{2}}} F(x+y) \prod_{j=1}^{m} E_{q_{1}, q_{2}}^{A_{\alpha_{j}}, B_{\alpha_{j}}}\left(\left\langle\alpha_{j}, x\right\rangle\right) d \mu(x) \\
& =T_{q_{1}}^{(1)}\left(T_{q_{2}}^{(1)}(F)\right)(y)
\end{aligned}
$$

for s-a.e. $y \in C_{a, b}[0, T]$.

Acknowledgments. The authors thank the referees for their helpful suggestions which led to the present version of this paper. 


\section{References}

[1] M. D. Brue, A functional transform for Feynman integrals similar to the Fourier transform, Thesis, Univ. of Minnesota, Minneapolis, 1972.

[2] R. H. Cameron and D. A. Storvick, An $L_{2}$ analytic Fourier-Feynman transform, Michigan Math. J. 23 (1976), no. 1, 1-30.

[3] S. J. Chang and D. M. Chung, Conditional function space integrals with applications, Rocky Mountain J. Math. 26 (1996), no. 1, 37-62.

[4] S. J. Chang, J. G. Choi, and D. Skoug, Integration by parts formulas involving generalized Fourier-Feynman transforms on function space, Trans. Amer. Math. Soc. 355 (2003), no. 7, 2925-2948.

[5] S. J. Chang and I. Y. Lee, A Fubini theorem for generalized analytic Feynman integrals and Fourier-Feynman transforms on function space, Bull. Korean Math. Soc. 40 (2003), no. $3,437-456$.

[6] D. M. Chung, Scale-invariant measurability in abstract Wiener spaces, Pacific J. Math. 130 (1987), no. 1, 27-40.

[7] T. Huffman, D. Skoug, and D. Storvick, A Fubini theorem for analytic Feynman integrals with applications, J. Korean Math. Soc. 38 (2001), no. 2, 409-420.

[8] _ Integration formulas involving Fourier-Feynman transforms via a Fubini theorem, J. Korean Math. Soc. 38 (2001), no. 2, 421-435.

[9] G. W. Johnson and D. L. Skoug, An $L_{p}$ analytic Fourier-Feynman transform, Michigan Math. J. 26 (1979), no. 1, 103-127.

[10] _ Scale-invariant measurability in Wiener space, Pacific J. Math. 83 (1979), no. 1, 157-176.

[11] E. Nelson, Dynamical Theories of Brownian Motion, Princeton University Press, Princeton, 1967.

[12] J. Yeh, Singularity of Gaussian measures on function spaces induced by Brownian motion processes with non-stationary increments, Illinois J. Math. 15 (1971), 37-46.

[13] , Stochastic Processes and the Wiener Integral, Marcel Dekker, Inc., New York, 1973.

IL YONG LEE

Department of Mathematics

DANKOOK UNIVERSITY

CheOnan 330-714, Korea

E-mail address: iylee@dankook.ac.kr

JAE GIL CHOI

Department of Mathematics

DANKOOK UNIVERSITY

CheOnan 330-714, Korea

E-mail address: jgchoi@dankook.ac.kr

SEung Jun Chang

Department of Mathematics

DANKOOK UNIVERSITY

Cheonan 330-714, Korea

E-mail address: sejchang@dankook.ac.kr 